This item was submitted to Loughborough's Research Repository by the author.

Items in Figshare are protected by copyright, with all rights reserved, unless otherwise indicated.

\title{
A novel multi-wavelength procedure for blood pressure estimation using opto-physiological sensor at peripheral arteries and capillaries
}

PLEASE CITE THE PUBLISHED VERSION

https://doi.org/10.1117/12.2287845

\section{PUBLISHER}

(c) 2018 Society of Photo-Optical Instrumentation Engineers (SPIE).

VERSION

VoR (Version of Record)

\section{LICENCE}

CC BY-NC-ND 4.0

\section{REPOSITORY RECORD}

Scardulla, Francesco, Sijung Hu, Leonardo D'Acquisto, Salvatore Pasta, Laura A. Barrett, Panagiotis Blanos, and Liangwen Yan. 2019. "A Novel Multi-wavelength Procedure for Blood Pressure Estimation Using Optophysiological Sensor at Peripheral Arteries and Capillaries”. figshare. https://hdl.handle.net/2134/33379. 


\section{A novel multi-wavelength procedure for blood pressure estimation using opto-physiological sensor at peripheral arteries and capillaries}

Francesco Scardulla, Sijung Hu, Leonardo D'Acquisto, Salvatore Pasta, Laura Barrett, et al.

Francesco Scardulla, Sijung Hu, Leonardo D'Acquisto, Salvatore Pasta, Laura Barrett, Panagiotis Blanos, Liangwen Yan, "A novel multi-wavelength procedure for blood pressure estimation using opto-physiological sensor at peripheral arteries and capillaries," Proc. SPIE 10486, Design and Quality for Biomedical Technologies XI, 1048614 (19 February 2018); doi: $10.1117 / 12.2287845$ 


\title{
A novel multi-wavelength procedure for Systolic blood pressure estimation using Opto-Physiological sensor at peripheral arteries and capillaries
}

\author{
Francesco Scardulla ${ }^{\mathrm{a}}$, Sijung Hu${ }^{*}$, Leonardo D'Acquisto ${ }^{\mathrm{a}}$, Salvatore Pasta $^{\mathrm{c}}$, Laura Barrett ${ }^{\mathrm{d}}$, \\ Panagiotis Blanos ${ }^{\mathrm{b}}$, Liangwen Yan ${ }^{\mathrm{e}}$
}

\author{
${ }^{a}$ DIID - Dipartimento dell'Innovazione Industriale e Digitale, Università degli Studi di Palermo, \\ Viale delle Scienze, Ed. 890128 Palermo, Italy; \\ ${ }^{\mathrm{b}}$ School of Electronic, Electrical and System Engineering, Loughborough University, Ashby Road, \\ Loughborough, Leicestershire, LE11 3TU UK; \\ ${ }^{\mathrm{c}}$ Fondazione Ri.MED, via Bandiera 11, 90134, Palermo, Italy; \\ ${ }^{\mathrm{d}}$ National Centre for Sport and Exercise Medicine, School of Sport, Exercise and Health Sciences, \\ Loughborough University, Ashby Road, Loughborough, Leicestershire LE11 3TU, UK; \\ ${ }^{\mathrm{e}}$ School of Mechatronic Engineering and Automation, Shanghai University, Shanghai 200072, \\ China;
}

\begin{abstract}
In this study, the Carelight multi-wavelength opto-electronic patch sensor (OEPS) was adopted to assess the effectiveness of a new approach for estimating the systolic blood pressure (SBP) through the changes in the morphology of the OEPS signal. Specifically, the SBP was estimated by changing the pressure exerted on an inflatable cuff placed around the left upper arm. Pressure acquisitions were performed both with gold standard (i.e. electronic sphygmomanometer), and Carelight sensor (experimental procedure), on subjects from a multiethnic cohort (aged $28 \pm$ 7). The OEPS sensor was applied together with a manual inflatable cuff, going slightly above the level of the SBP with increases of $+10 \mathrm{mmHg}$ and subsequently deflated by $10 \mathrm{mmHg}$ until reaching full deflation. The OEPS signals were captured using four wavelength illumination sources (i.e., green $525 \mathrm{~nm}$, orange $595 \mathrm{~nm}$, red $650 \mathrm{~nm}$ and IR $870 \mathrm{~nm}$ ) on three different measuring sites, namely forefinger, radial artery and wrist. The implemented algorithm provides information on the instant when the SBP was reached and the signal is lost since the vessel is completely blocked. Similarly, it detected the signal resumption when the external pressure dropped below the SBP. The findings demonstrated a good correlation between the variation of the pressure and the corresponding OEPS signal with the most accurate result achieved in the fingertip among all wavelengths, with a temporal identification error of $8.07 \%$. Further studies will improve the clinical relevance on a cohort of patients diagnosed with hyper- or hypotension, in order to develop a wearable blood-pressure device.
\end{abstract}

Keywords: Multi-Wavelength, blood pressure, systolic blood pressure, opto-electronic patch sensor (OEPS), wearable sensor, photopletismgraphy, PPG

Design and Quality for Biomedical Technologies XI, edited by Ramesh Raghavachari, Rongguang Liang, Proc. of SPIE Vol. 10486, $1048614 \cdot$ ? 2018 SPIE · CCC code: 1605-7422/18/\$18 · doi: 10.1117/12.2287845 


\section{INTRODUCTION}

\subsection{BACKGROUND}

In the last decades, both the world population and life expectancy have been increasing dramatically. These two trends have led to a subsequent increase in the number of people falling sick and in need of health assistance in specialized centers. This epidemiological scenario affecting the industrialized countries bears considerable consequences on the organization and costs of health systems. This phenomenon might further spread in the future with negative economic effects, unless strategies are designed focusing primarily on preventing those conditions which lead to chronic diseases. Among these disorders, cardiac failure affects approximately 6.5 million Europeans and is a major cause of hospitalizations. The first edition of European Cardiovascular Disease Statistics states that costs due to cardiovascular diseases are estimated in $€ 210$ billion per year, thereby leading to a remarkable healthcare burden for the economy of the European countries. One of these diseases, affecting 6.5 million Europeans, is cardiac failure, which leads to a high number of hospitalizations and thus represents the main expense for international and European health systems, as shown in figure 1 (Socio-Economic impact of mHealth. An assessment report for European Union, Pwc 6-2013). The use of sensors might represent a solution to this problem, as they are able to monitor the physical condition of patients, thus identifying signs of disease deterioration, which are not perceived as such by the patient. An immediate response by health practitioners might prevent a worsening of the patient condition, thus resulting in lower hospitalization rates and ultimately in lower costs.

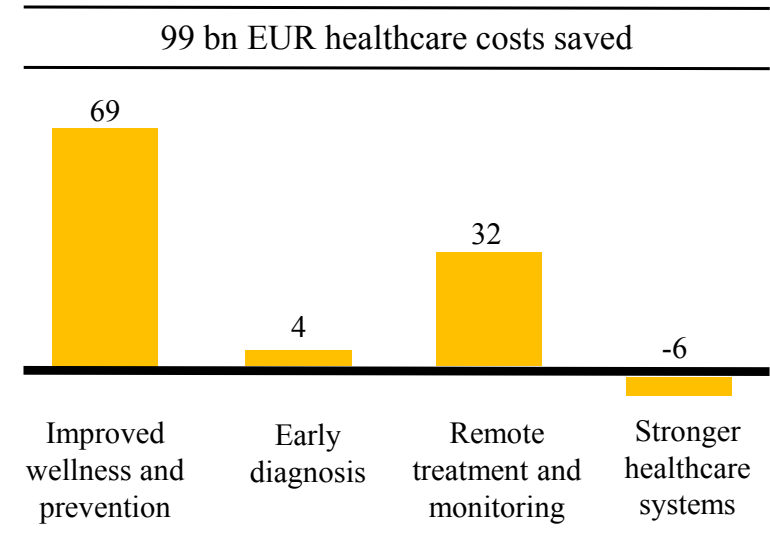

Figure (1) Healthcare costs saved in EU (adapted from: HOPE, European hospital and healthcare federation, PwC analysis)

Wearable sensors, more specifically, can increase the effectiveness of medical equipment, such as life-supporting implants, and can enable remote monitoring of vital signs and other health factors such as pressure, heart rate, heart rate variability, sweat, body temperature, movement and blood oxygenation. This clinical information can be used to monitor and prevent numerous diseases as well as inappropriate behaviors. Specifically, blood pressure (BP), is one of the most basic vital signs and provides physicians with important metrics regarding the patient's baseline cardiovascular status 
with both clinical and sports applications. However, BP monitoring devices currently available on the market are rather uncomfortable for wearable purposes.

Photoplethysmography (PPG) perfectly serves this purpose, as it is a low-cost and reliable technology, which has been used for decades in the field of medical devices. It enables the recording of information related to volume variations within the cardiovascular system and consequently to cardiac activity $[1,2]$ such as cardiac frequency, blood oxygenation and heart rate variability. Previous studies have described the measurement of blood pressure using the pulse transit time. Nevertheless, this technique is not suitable for a wearable device, since it requires measurement recalibration and the accuracy is influenced by several factors [3]. Furthermore, the use of PPG technology is still challenging because of the absorption variability in the intensity of the LED light in each patient, due to both ethnicity (e.g. melanin, tissue constitution, connective tissue properties) and variations within the same ethnic group (e.g. body and skin hydration, fat percentage, tattoos, age). For these reasons, the PPG signal heavily relies on the penetration depth of the light beam [4] and is often the result of the combination of volume variations measured at different depths.

We sought to determine the effectiveness of a new approach to detect the change in the morphology by using a customized opto-electronic patch sensor (OEPS) based on the PPG technology and equipped with four different waveleghts (i.e. $525 \mathrm{~nm}, 595 \mathrm{~nm}, 650 \mathrm{~nm}$ and $870 \mathrm{~nm}$ ). The aim was to determine the systolic blood pressure (SBP) by changing the pressure exerted on an inflatable cuff placed smoothly and snugly around the left upper arm of subjects from a multiethnic cohort. Generally, the window chosen for optical measurement ranges from 405 to $1064 \mathrm{~nm}$ [5] and varies depending on the application, which involves three different human skin layers (i.e. epidermis, dermis and subcutaneous). Using four wavelengths simultaneously and covering a range between 525 and $870 \mathrm{~nm}$, we aimed at understanding more in depth how different lights interact within biological tissues, as the blood flow determined by the manually inflated air-cuff changes. Finally, we sought to identify both the interruption and the resumption of the blood flow, assessing which wavelength is more effective on a given body site, depending on the physical features of the subject.

\section{METHODODOLOGY}

\subsection{WORKING PRINCIPLES OF THE OPTO-ELECTRONIC PATCH SENSOR}

Like most of the non-invasive miniaturized wearable devices for physiological measurements, the OEPS was designed to be applied on the skin surface at different sites of the human body, such as palm and wrist. Blood-vessel volume changes can be continuously acquired in the peripheral circulation, thus reflecting the cardiac activity and therefore providing considerable information of cardiovascular physiology (i.e. heart rate, respiration rate, heart rate variability, blood oxygenation).

The basic principle behind the OEPS technique is related to the fact that, when a certain light beam travels through biological tissues, it is absorbed by the whole involved tissue including skin pigments, venous and arterial blood. The same light is also reflected back to the photo-detector sensor, which can identify the changes in the intensity of the light (i.e. voltage). A standard signal shows a continuous component (DC) that is determined by the non-pulsatile part of the tissue and an alternating component (AC) that is the one reflecting the changes in blood amount within the vessels. Since blood absorbs the light more than the other surrounding tissues, the AC component can be conveniently superimposed on the DC baseline and synchronized to the heartbeats. 


\subsection{OEPS DESIGN}

The OEPS is a wearable multi-channel sensor [6] that operates in reflectance mode (both LEDs and the photodetector are fixed to the same plane). The sensor has a square shape (i.e. $18 \mathrm{~mm} \times 18 \mathrm{~mm}$ ) of $0.1 \mathrm{~mm}$ thickness, making it particularly suitable for skin applications without causing any discomfort to the subjects. A Si-photodetector (PD) is positioned in the middle of a flexible patch with a total area of $1.69 \mathrm{~mm}^{2}$ (Hamamatsu photonics K. K., Hamamatsu City, Japan). The PD is surrounded by 16 light-emitting diodes (LEDs, JMSIENNA Co., Ltd, TouFen, Taiwan) placed in a cross configuration at a specific distance to avoid the saturation of the signal from a closer source as shown in figure 2. Specifically, there are four LEDs for each wavelength (i.e., green $525 \mathrm{~nm}$, orange $595 \mathrm{~nm}$, red $650 \mathrm{~nm}$ and IR $870 \mathrm{~nm}$ ). The wavelength of each LED group increases progressively from the center (i.e. PD) outwards.
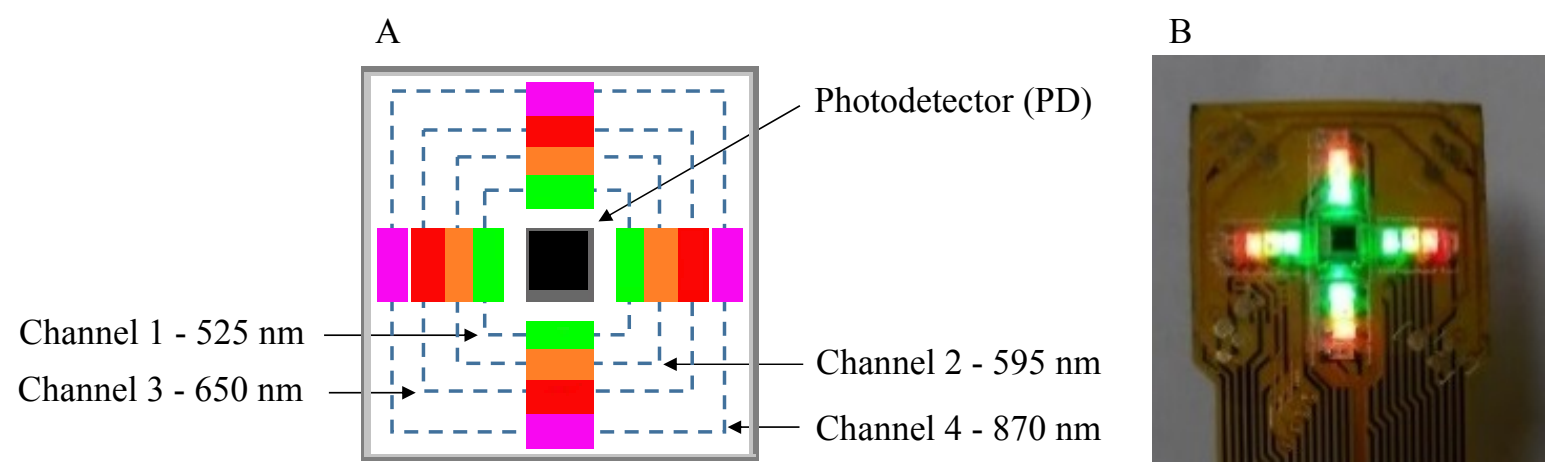

Figure (2) Schematic diagram of OEPS (A). Every channel includes four LEDS and has different wavelength, green, orange, red and infrared respectively. Image of OEPS with all the 16 LEDs active (B).

A transparent thin film layer of clear epoxy medical adhesive was placed on top of the patch sensor to protect the optical components, to prevent direct skin contact and to avoid optical interference due to sweating.

The block diagram shown in Figure 3 summarizes the general architecture of the OEPS system, including a) the Patch Sensor, b) the electronic board (DISCO4, Dialog Devices Ltd., Reading, Berkshire, UK) consisting of an Analog Front End (AFE) and the Digital Signal Processing (DSP), c) the Microprocessor Unit (MCU), d) the Multifunction Data Acquisition USB Device (DAQ, USB-6009, National Instruments Co., Novato, CA, USA) and e) the control software of PPG board operated using LabVIEW GUI (National Instruments Co., USA). A pre-amplifier, a Multiplexer (MUX), a differential amplifier and a band-pass filter BPF were adopted to build the AFE. 


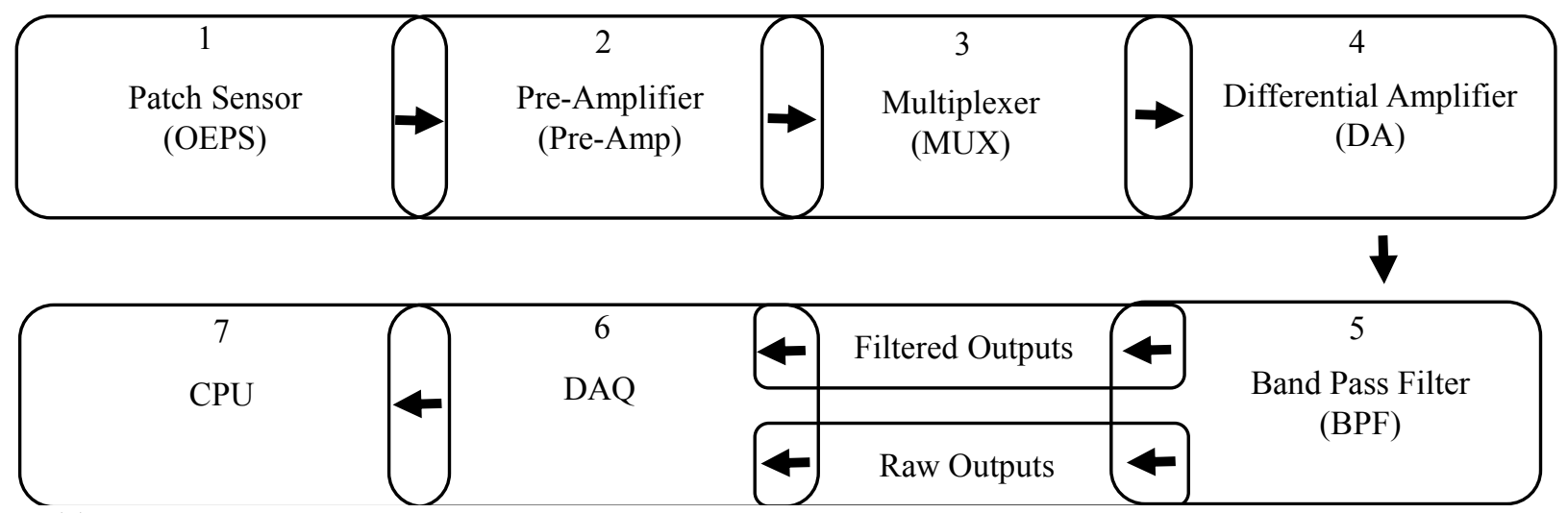

Figure (3) Schematic block diagram of the system: the Analogue Front End (AFE) includes blocks 2, 3, 4 and 5, a multifunction Data Acquisition USB Device (block 6) and a computer (block 7).

The AFE is a set of analog signal conditioning circuits that use sensitive analog amplifiers, operational amplifiers, filters, and application-specific integrated circuits for sensors to provide a configurable and flexible electronical functional block. This is needed to interface the OEPS to the microcontroller. The amplifier is designed to increase the amplitude of the weak signal, because the AC component of the signal is almost 2-5\% of the DC component [7]. The following stage is the multi- and de-multiplexer, where each wavelength of the signal is individually separated to provide only a single wavelength LED illumination at a time in the next stages. It also allows the PD not to produce errors while synchronizing it with the specific wavelength received at a certain time. Then, the signal flows to the Band Pass Filter (BPF) where the frequency is cut off between 0.2 and $30 \mathrm{~Hz}$ to prevent the signal from being contaminated by external noise sources, such as ambient light, power supply, electromagnetic and electronical interferences. During all acquisition procedures, a specific functionality of the system has been used to enable the automatic adaptation of the LED intensity. Therefore, due to the variability in the tissue properties of the subject (i.e. skin pigmentation, fat thickness, hydration, tattoos), the system takes advantage of two different thresholds, the lower and the upper one, within which the optimal range of light intensity is automatically set. This was done to produce the right illumination intensity and allows to achieve a good AC component of the signal in all circumstances, even in those subjects whose tissue characteristics would not be optimal for an optical investigation. Finally, the signal is digitalized and each channel is recorded and stored simultaneously.

\subsection{PHYSIOLOGICAL MONITORING PROTOCOL}

Twenty-five healthy subjects from a multiethnic cohort (aged $28 \pm 7$ ) were enrolled for the data acquisition. None of the enrolled subjects took stimulants or drugs that could have influenced blood pressure measurements. All the acquisitions were collected with the subjects at rest at a room temperature ranging between $22^{\circ} \mathrm{C}$ and $26^{\circ} \mathrm{C}$. Before the acquisitions, the skin colors were classified in six groups by using the Fitzpatrick and Von Luchan's scale, which ranges from 1 (very light color) to 6 (very dark color). Participants were in sitting position and the PPG signals were captured with the 4 wavelength illumination sources (i.e., green, orange, red and IR).

The OEPS was positioned away from any scar, tattoo or skin blotch and a constant pressure was applied between the sensor probe and the skin site [8]. SBP acquisitions were performed with both gold standard (standard procedure), that is measuring blood pressure using an automatic sphygmomanometer (Omron blood pressure meter, M6 comfort Omron inc, Chicago, IL, USA), and OEPS sensors (experimental procedure), in which subjects wore both an inflatable cuff and 
the OEPS sensor. More specifically, during the experimental procedure, the cuff was inflated manually with increases of $+10 \mathrm{mmHg}$ every 10 seconds, going slightly above the level of the SBP previously detected by the gold standard device and was subsequently deflated by $10 \mathrm{mmHg}$ every 10 seconds until reaching full deflation. The OEPS signal was measured in three different sites (i.e. fingertip, radial artery and wrist) as shown in Figure 4 and before each of the three measurements with the experimental procedure the pressure was recorded with the standard procedure as reference. A two-minute break was scheduled in between measurements. This human protocol was approved by the Loughborough University Ethics Committee.

Standard Procedure

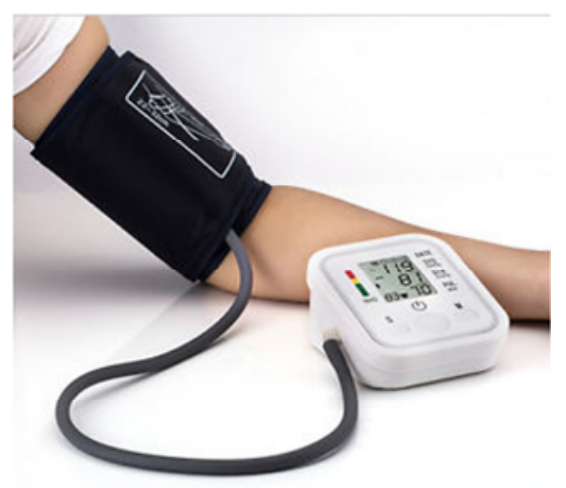

Experimental Procedure

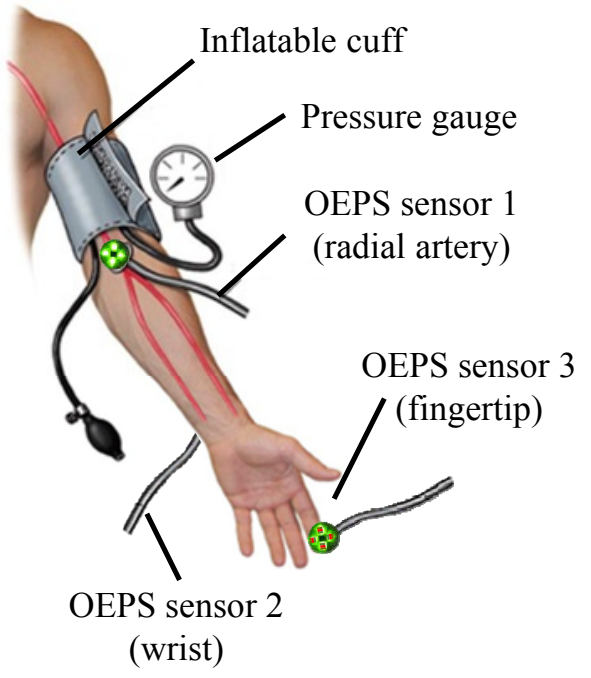

Figure (4) Standard (A) and experimental procedure (B)

When the air was injected into the cuff, the blood vessel was gradually blocked by the increasing pressure of the cuff. Close to the systolic pressure, the OEPS signal became too weak for the sensor to pick it up. Upon its disappearance, the cuff pressure was decompressed gradually. Then, the OEPS pulse re-appeared at a certain point increasing its amplitude. Data were acquired by the use of LabVIEW (National Instrument, Austin, Texas, U.S.) and recorded with a frequency of $256 \mathrm{~Hz}$ and then analyzed off-line using an algorithm in MatLab, (MathWorks, Inc., Natick, Massachusetts, U.S.) specifically developed for the experiment.

The MatLab algorithm was used to analyze the OEPS signal at all the wavelengths for each sensor site, taking into account the slope, the angles, the fitting curves of all peaks and integrals as well as the areas. It provides approximations on the instant at which the systolic pressure was reached during the cuff inflation and therefore the loss of the signal, as well as the time in which it returned again below the systolic pressure and therefore the signal was resumed. The pressure value recorded at that instant within the manually-inflated cuff was compared to that recorded by the gold standard sphygmomanometer.

Moreover, the algorithm was able to identify all the peaks and to provide, among other things, the time of signal loss and reacquisition, thus allowing for a correlation with the pressure inside the inflatable air cuff.

Figure 5 shows the signal vs time profile provided by Matlab, which offers several information including the two instants in which the pressure went above and below the systolic pressure. This value was subsequently correlated to the SBP 
previously measured with the gold standard device. The code was designed to apply an adaptive filter, whose coefficients vary depending on the signal quality. Further processing analyses were carried out by means of electronic data sheet.

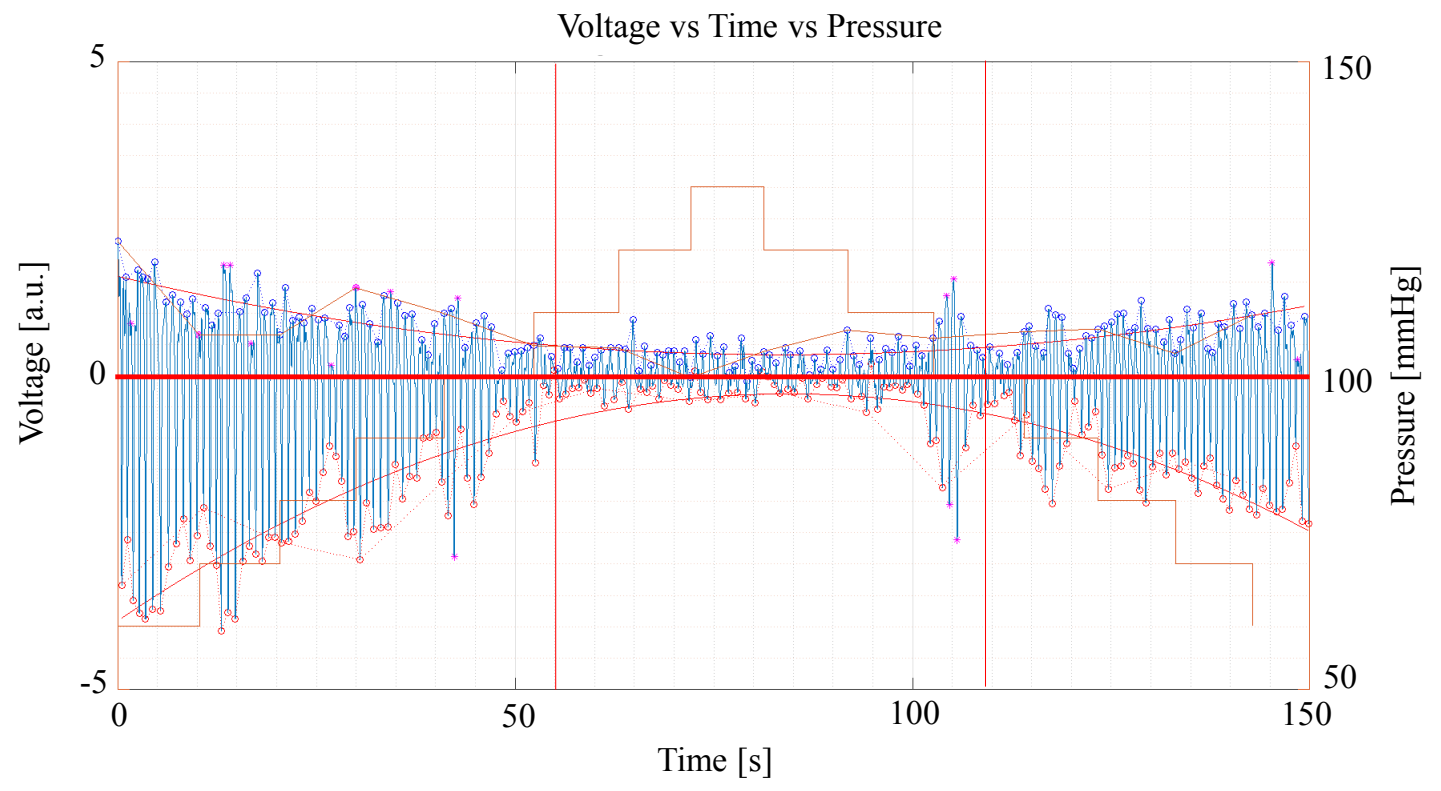

Figure (5) Green light processed with MatLab from an OEPS signal measured in the fingertip. The BP of the subject recorded with the standard procedure was $110 / 60[\mathrm{~mm} / \mathrm{Hg}]$

\section{PRELIMINARY RESULTS AND DISCUSSION}

In this preliminary study, the general pattern described by the OEPS (Figure 6) showed a good correlation between the pressure variation that is expected during the inflation and deflation of the cuff and the corresponding OEPS signal. This is more evident for subjects with a skin classification color between I and III, but less apparent in subjects whose skin color ranges from IV to VI. This results led us to implement a further mathematical function to recognize the quality of the signal from the onset, in terms of attenuation percentage, and to adapt the Savitzky-Golay filter coefficient so as to identify the instants of signal loss and reacquisition better. Despite the use of the adaptive filter, the skin-colored subjects ranging from IV to VI provided lower quality results for all wavelengths. 

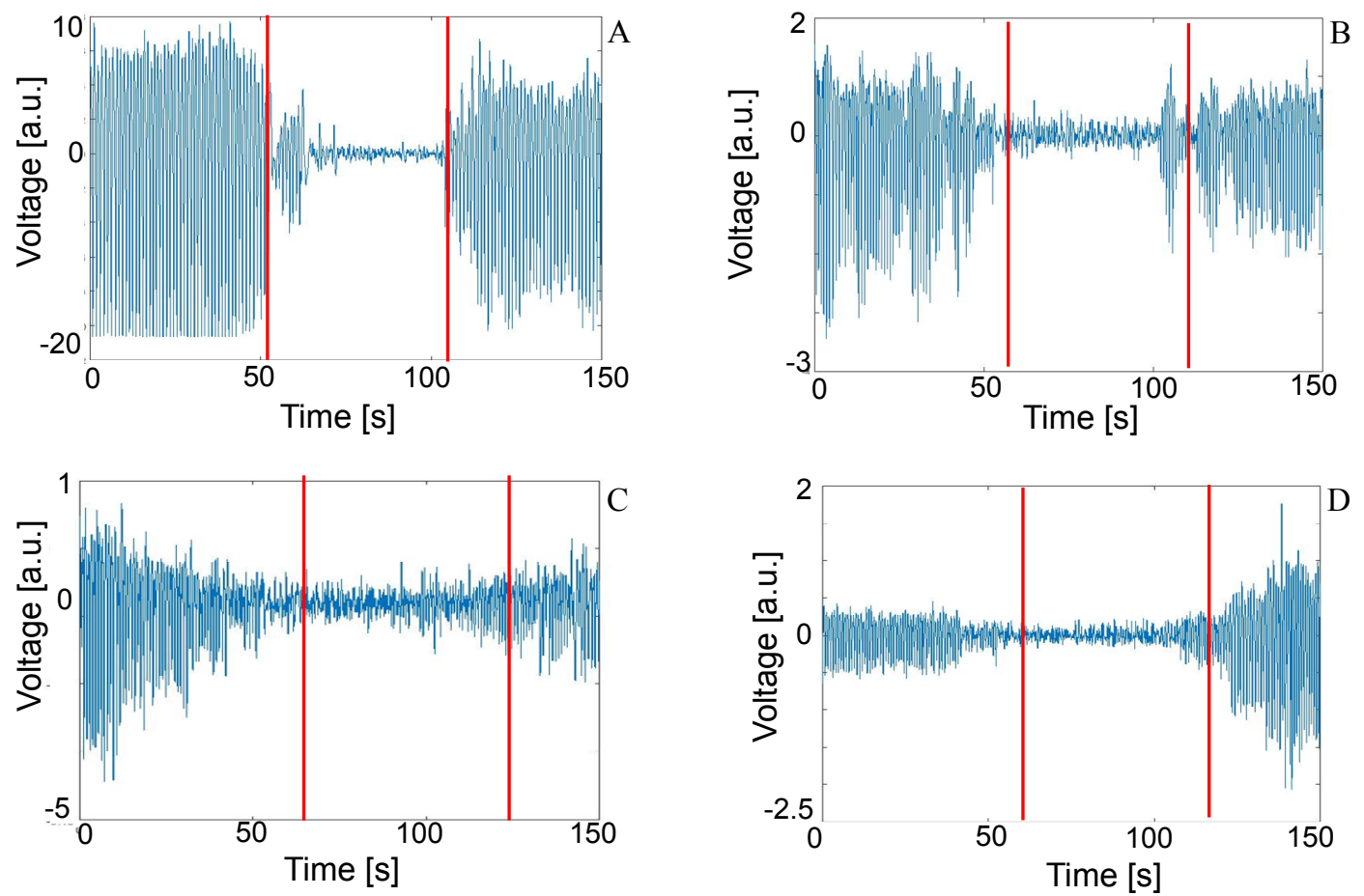

Figure (6) Races signal comparison at fingertips of four different subjects by using green light source. The skin types are II (figure A), III (figure B), VI (figure C) and IV (figure D)

Table 1 summarizes the skin color classification of the subjects, as well as the average SBP measurements achieved with the gold standard device. Average systolic pressure values of the subjects corresponded to the physiological values of a healthy subject.

Table 1: Gold standard device Systolic Blood Pressure obtained before performing the experimental acquisition in the three different sites, which are fingertip (SBP 1), radial artery (SBP 2) and wrist (SBP 3)

\begin{tabular}{lccccc}
\hline Skin classification & $\mathrm{N}^{\circ}$ of subjects & Age & $\begin{array}{c}\mathrm{SBP} 1 \\
{[\mathrm{~mm} / \mathrm{Hg}]}\end{array}$ & $\begin{array}{c}\mathrm{SBP} 2 \\
{[\mathrm{~mm} / \mathrm{Hg}]}\end{array}$ & $\begin{array}{c}\mathrm{SBP} 3 \\
{[\mathrm{~mm} / \mathrm{Hg}]}\end{array}$ \\
\hline \hline I (very light) & 1 & 23 & 143 & 125 & 126 \\
II (light) & 9 & $26 \pm 4$ & $119 \pm 11$ & $115 \pm 14$ & $119 \pm 15$ \\
III (light intermediate) & 11 & $28 \pm 9$ & $113 \pm 10$ & $109 \pm 11$ & $110 \pm 8$ \\
IV (dark intermediate) & 1 & 32 & 114 & 106 & 103 \\
V (dark) & 2 & $30 \pm 2$ & $103 \pm 3$ & $100 \pm 2$ & $105 \pm 1$ \\
VI (very dark) & 1 & 44 & 119 & 142 & 130 \\
\hline \hline
\end{tabular}


Preliminary results also demonstrated that the fingertip site provided the most accurate values among all wavelength average results, with an error in the identification of the temporal window of $8.07 \%$ when compared to the radial artery (error of $13.17 \%$ ) and the wrist (17.44\%).

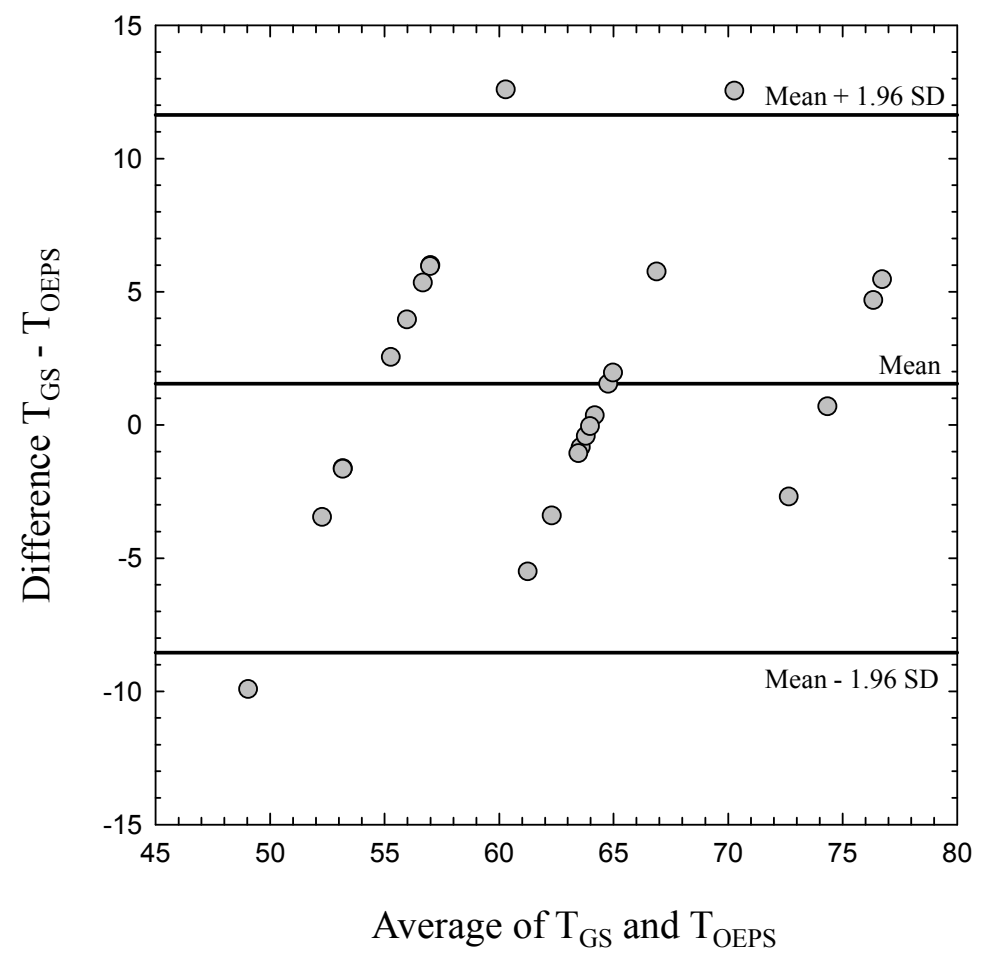

Figure (7) Bland-Altman plot describing the agreement between time matching for values extracted from both gold standard and experimental methods from green light in fingertip site.

In the same skin site, the green light offered the best performance in the identification of the 10-second temporal window when the systolic pressure occurred during the cuff inflation. A Bland-Altman analysis demonstrated the high agreement between gold standard and experimental measurements (Figure 7).

The green light recorded the best performance for every site, followed by the orange one with a slight error difference of $2 \%$. The red light obtained the best results on the fingertip, with an error of $6.33 \%$, as well as the infrared with an error of $7.27 \%$, which has always shown the worst results when compared with the other wavelengths.

In addition, better results were achieved in the inflation phase than during the deflation of the cuff, since some delay was observed in the recovery of the bloodstream in the artery and therefore in the regeneration of the signal.

As Liu J. et al suggest [4], the multi-wavelength PPG approach relies on the selection of the wavelength combination and the reference signal. This approach can enable a better depth sensitivity in optical measurements based on a multidistance approach. Indeed, if we consider biological tissues as a set of optical media, it can describe the interaction 
between light and tissue assessing an opto-physiological model that could represent a good tool to identify vital parameters such as SBP better.

The multi-wavelength opto-electronic patch sensor (OEPS), through the implementation of a specific protocol and dedicated algorithm, has proven to detect the disappearance of the signal effectively and correlate it with the SBP. Moreover, the automatic adaptation of the LED intensity improved the performance of the sensor, allowing an objective and automatic design independent from the user.

Recent studies $[9,10]$ have shown that green light illumination source has greater modulation with regards to different skin types during the heart rate detection and moreover minimizes motion artifacts [11]. We achieved similar results in the detection of the SBP (i.e., signal attenuation), as the green light performed better than all the other measurement sites. However, the optimal wavelength for each skin place may be different in other approaches due to signal intensity, quality and tissue properties.

\section{CONCLUSION AND FUTURE WORK}

In this study we move a step closer to the designing of an effective a multi-wavelength, PPG-based OEPS sensor, with a view to estimate the systolic blood pressure through the changes in the morphology of the OEPS signal by changing the pressure exerted on an inflatable cuff placed around the left upper arm. The effectiveness of this methodology was assessed by performing a protocol on subjects from a multiethnic cohort of 25 subjects with different skin colors. The automatic adaptation of the LED intensity provided an optimal illumination for any skin and tissue characteristics of subjects. The comparison between gold standard and experimental procedure showed the reliability and effectiveness of the proposed approach. The Bland-Altman analysis demonstrated high agreement of the OEPS signal versus the goldstandard sphygmomanometer, with green light exhibiting more accurate blood-pressure measurements among all the sites.

The versatility of the system allowed the implementation of the proposed approach for monitoring devices aimed at measuring cardiovascular parameters such as SBP. Further studies will improve the clinical significance on a patients cohort diagnosed with hyper- or hypotension in order to develop a device that may be used in wearable blood-pressure devices.

\section{ACKNOWLEDGMENT}

Authors would like to thank the Loughborough University for its support. Francesco Scardulla thanks the University of Palermo for supporting his research.

\section{REFERENCES}

[1] Challoner, A. "Photoelectric plethysmography for estimating cutaneous blood flow," Phys. Med. Biol. 19(3), 317-28 (1974).

[2] Allen, J. "Photoplethysmography and its application in clinical physiological measurement," Physiol. Meas. 28(3), R1-R39 (2007). 
[3] Peter, L., Noury, N., Cerny, M., "A review of methods for non-invasive and continuous blood pressure monitoring: Pulse transit time method is promising?" IRBM 35, 271-282 (2014).

[4] Liu, J., Yan, B.P.-Y., Dai, W.-X., Ding, X.-R., Zhang, Y.-T., Zhao, N, "Multi-wavelength photoplethysmography method for skin arterial pulse extraction," Biomed. Opt. Express. 7(10), 4313-4326 (2016).

[5] Spigulis, J., Gailite, L., Lihachev, A., Erts, R., "Multi-wavelenght photoplethysmography for simultaneous recording of skin blood pulsations at different vascular depths," Applied Optics 46, 1754-1759 (2007).

[6] Hu, S., Azorin-Peris, V., "Opto-Physiological sensor and method of assembly," WO 2015056007 A1, April 23 2015.

[7] Meglinski, I.V., Matcher, S.J., "Computer simulation of the skin reflectance spectra," Comput. Meth. Program. Biomed. 70, 179-186 (2003).

[8] Teng X.F., Zhang, Y.T., "Theoretical study on the effect of sensor contact force on pulse transit time," IEEE Trans, Biomed. Eng. 54 (8), 1490-1498 (2007).

[9] Fallow, B.A., Tarumi, T., Tanaka, H., "Influence of skin type and wavelength on light wave reflectance," J. Clinic. Monitor. Comput. 27, 313-317 (2013).

[10] Yan, L., Hu, S., Alzahrani, A., Alharbi, S., Blanos, P., “A multi-wavelength opto-electronic patch sensor to effectively detect physiological changes against human skin types," Biosensors. 7(2), 22 (2017).

[11] Tamura, T., Maeda, Y., Sekine, M., Yoshida, M. "Wearable photoplethysmography sensor-past and present," Electronics. 3, 282-302, (2014). 